\title{
Deficits of entropy modulation of the EEG: A biomarker for altered function in schizophrenia and bipolar disorder?
}

\author{
Vicente Molina, MD, PhD; Alba Lubeiro, PhD; Rodrigo de Luis Garcia, PhD; \\ Javier Gomez-Pilar, PhD; Oscar Martín-Santiago, MD, PhD; María Iglesias-Tejedor, MD; \\ Pedro Holgado-Madera, MD; Rafael Segarra-Echeverría, MD, PhD; \\ María Recio-Barbero, MSc; Pablo Núñez, MSc; Mahmoud Karim Haidar, MD; \\ Jessica Fernández-Sevillano, MSc; Javier Sanz-Fuentenebro, MD, PhD
}

\begin{abstract}
Background: The synchronized activity of distributed neural assemblies - reflected in the electroencephalogram (EEG) - underpins mental function. In schizophrenia, modulation deficits of EEG spectral content during a P300 task have been replicated. The effects of treatment, chronicity and specificity in these deficits and their possible relationship with anatomic connectivity remain to be explored. Methods: We assessed spectral entropy modulation of the EEG during a P300 task in 79 patients with schizophrenia (of those, 31 were in their first episode), 29 patients with bipolar disorder and 48 healthy controls. Spectral entropy values summarize EEG characteristics by quantifying the irregularity of spectral content. In a subsample, we calculated the network architecture of structural connectivity using diffusion tensor imaging and graph-theory parameters. Results: We found significant spectral entropy modulation deficits with task performance in patients with chronic or first-episode schizophrenia and in patients with bipolar disorder, without significant pre-stimulus spectral entropy differences. The deficits were unrelated to treatment doses, and spectral entropy modulation did not differ between patients taking or not taking antipsychotics, lithium, benzodiazepines or antidepressants. Structural connectivity values were unrelated to spectral entropy modulation. In patients with schizophrenia, spectral entropy modulation was inversely related to negative symptoms and directly related to verbal memory. Limitations: All patients were taking medication. Patients with bipolar disorder were euthymic and chronic. The cross-sectional nature of this study prevented a more thorough analysis of state versus trait criteria for spectral entropy changes. Conclusion: Spectral entropy modulation with task performance is decreased in patients with schizophrenia and bipolar disorder. This deficit was not an effect of psychopharmacological treatment or structural connectivity and might reflect a deficit in the synchronization of the neural assemblies that underlie cognitive activity.
\end{abstract}

\section{Introduction}

Mental functions are underpinned by the quickly evolving synchronization of distributed neural assemblies. ${ }^{1,2}$ Because the electroencephalogram (EEG) reflects this synchronized activity, its modulation during cognitive activity can be used as a proxy in analyses of the substrates of mental function. Given the swiftly evolving synchrony of these neural assemblies, ${ }^{3,4}$ the high temporal resolution of EEG also makes it a suitable tool for such analyses. Therefore, assessments of EEG modulation with cognition are of potential interest when studying the substrates of alterations in mental function, such as those found in psychoses.
The EEG data are frequently analyzed at individual sensors in different spectral bands, resulting in many comparisons between cognitive conditions and groups. A solution to this problem is the assessment of parameters that summarize EEG characteristics, such as spectral entropy, which quantifies the signal degree of uncertainty ${ }^{5}$ : high spectral entropy corresponds to more uniform distribution of spectral content (a highly random signal), whereas low spectral entropy indicates a spectrum whose power is condensed to a narrower frequency range (a more regular signal). Differences between patients and controls in EEG modulation with cognition can be assessed by comparing spectral entropy values between the windows immediately before

Correspondence to: V. Molina, Department of Psychiatry, School of Medicine, University of Valladolid. Av. Ramón y Cajal, 7, Valladolid 48005, Spain; vicente.molina@uva.es

Submitted Feb. 14, 2019; Revised Sep. 30, 2019; Accepted Nov. 17, 2019; Published online Feb. 26, 2020

DOI: $10.1503 /$ jpn. 190032 
and after a target stimulus in a task. Because variations in task performance or collaboration can lead to different patterns of EEG modulation, it seems important that the cognitive load of the task does not preclude adequate levels of collaboration or performance on the part of patients. The P300 task, which involves attention and working memory, fulfills these requirements and has been widely used in the field.

In previous reports with low-density EEG recordings, ${ }^{6}$ we showed a deficit in the spectral entropy modulation of EEG during a P300 task in patients with schizophrenia compared to healthy controls. We replicated this finding in a different and larger sample, suggesting a possible role for spectral entropy modulation as a biomarker in schizophrenia, ${ }^{7}$ and we further described the architecture of functional connectivity that underlies this deficit using graph theory. ${ }^{8}$ In controls, task-related spectral entropy modulation was associated with the slowing of EEG rhythms during task response, and a relative increase in $\theta$ power. ${ }^{6,7}$ These changes were smaller in patients, suggesting altered synchronization of underlying neural assemblies in schizophrenia.

The specificity of this deficit (i.e., whether similar deficits are found in other psychoses) and its possible relationship with treatment and chronicity remain to be clarified. Furthermore, it is not known whether the deficit relates to the structural connectivity alterations reported in schizophrenia. ${ }^{9}$ This possibility is supported by the associations reported between structural connectivity values and modulation of the EEG network in schizophrenia. ${ }^{10}$ Nevertheless, because functional connectivity exists between regions without direct anatomic connection, ${ }^{11}$ the possibility that EEG modulation with cognition is unrelated to alterations in structural connectivity cannot be discarded.

In the present study, we addressed these questions using a higher-density EEG in a new sample including patients with chronic and first-episode schizophrenia, patients with bipolar disorder and healthy controls. We obtained EEG and diffusion tensor imaging (DTI) data to assess bioelectrical modulation and structural connectivity architecture, respectively. Our hypotheses were that in the present sample, (i) spectral entropy modulation would be decreased in patients with schizophrenia compared to patients with bipolar disorder and healthy controls, because decreases in spectral entropy modulation have been found in patients with chronic and first-episode schizophrenia, ${ }^{7}$ as well as in patients with schizophrenia who have received only minimal treatment ${ }^{6}$; (ii) deficits in spectral entropy modulation would be unrelated to antipsychotic therapy or other types of treatment, because we showed in a smaller sample that current antipsychotic doses were unrelated to spectral entropy modulation ${ }^{7}$ and that a single dose of haloperidol did not change significantly spectral entropy modulation in healthy volunteers ${ }^{6}$; and (iii) spectral entropy modulation would be associated with structural connectivity architecture, given our previous finding of an association between the graph parameters of DTI-based structural networks and modulation of the graph parameters of the functional EEG network. ${ }^{10}$

\section{Methods}

\section{Clinical and cognitive assessment}

We recruited 156 participants: 79 patients with schizophrenia (including 31 with first-episode schizophrenia), 29 euthymic patients with bipolar disorder type I and 48 healthy controls. We selected the controls from a database of 127 people with EEGs identically recorded and analyzed. The euthymic state was defined as scores lower than 8 on the Hamilton Depression Rating Scale ${ }^{12}$ and lower than 5 on the Young Mania Rating Scale, ${ }^{13}$ in the absence of an affective episode for at least the last 3 months.

Electroencephalogram, clinical and cognitive data were available for all participants. Diffusion tensor imaging data were available for 21 patients with chronic schizophrenia, 18 patients with first-episode schizophrenia, 21 patients with bipolar disorder and 20 healthy controls. Data from 24 patients with schizophrenia and 34 healthy controls in the present study were included in a previous report. ${ }^{7}$

Patients were diagnosed by one of the psychiatrists in the group according to the criteria of the Diagnostic and Statistical Manual of Mental Disorders, 5th edition. Symptoms were scored using the Positive and Negative Syndrome Scale. ${ }^{14}$ Depression and mania were scored using the Hamilton Depression Rating Scale ${ }^{12}$ and the Young Mania Rating Scale. ${ }^{13}$ Exclusion criteria for patients and controls were history of neurologic illness (including cranial trauma with loss of consciousness), past or present substance abuse (except nicotine or caffeine), and IQ lower than 70. In addition, controls were excluded if they had any current psychiatric diagnosis or treatment. Healthy controls were recruited through newspaper advertisements.

We collected cognitive data using the Wechsler Adult Intelligence Scale (3rd edition), ${ }^{15}$ the Wisconsin Card Sorting Test (percentage of perseverative errors) ${ }^{16}$ and the Spanish version of the Brief Assessment of Cognition in Schizophrenia. ${ }^{17}$ We assessed social cognition using the Meyer, Salovey and Caruso Emotional Intelligence Test. ${ }^{18}$

All participants provided written informed consent after reviewing full printed information. The ethical committees of the participating hospitals approved the study.

\section{EEG recording}

We acquired EEG recordings using a 32-channel device (BrainVision, Brain Products GmbH) following the 10-20 system. Impedance was kept under $5 \mathrm{k} \Omega$. The EEG sampling frequency was $500 \mathrm{~Hz}$, and the $\mathrm{Cz}$ electrode was used as the reference. Participants performed a 3-tone auditory oddball task (P300 task). This task elicits well-known cognitive activity and event-related potentials with identified spatial distribution. Given the relatively low demands of this task, patient collaboration is usually good, making it adequate to test our hypothesis that a hampered task-related modulation of cerebral activity contributes to cognitive deficits in psychoses.

The auditory oddball task consisted of a 3-tone auditory oddball paradigm randomly presented: target $(500 \mathrm{~Hz}$ tone, 
probability 0.2$)$, distractor $(1000 \mathrm{~Hz}$ tone, probability 0.2$)$ and standard (2000 Hz tone, probability 0.6). The task lasted 13 minutes, during which participants remained seated with their eyes closed and were instructed to press a mouse button immediately after the target tones. For subsequent analyses, we considered only the target tones followed by a button press.

We re-referenced the EEG recordings to the average activity of all active sensors. ${ }^{19}$ We used a finite impulse response band-pass filter $(1-70 \mathrm{~Hz})$, and a notch filter to remove power line interference $(50 \mathrm{~Hz})$. Next, we conducted a 3-step procedure to reject artifacts ${ }^{20,21}$ : independent component analysis to remove eye-blinks and muscle artifacts ${ }^{22}$; trial segmentation into $1 \mathrm{~s}$ windows, ranging from $300 \mathrm{~ms}$ before to $700 \mathrm{~ms}$ after the stimulus onset; and adaptive trial rejection using statistical thresholding to discard noise trials. ${ }^{21}$

\section{EEG spectral analysis}

Electroencephalogram recordings are non-stationary signals whose characteristics change over time, so non-stationary signal analysis techniques are appropriate for describing their changing properties. ${ }^{23,24}$ In the present study, we used continuous wavelet transform (CWT) to obtain the timefrequency representation. The CWT is well-suited to the study of EEG, because it provides a good balance between time and frequency resolution. ${ }^{25}$ We selected the complex Morlet wavelet as the mother wavelet, because it provides a biologically plausible fit for EEG signals. ${ }^{25}$ We obtained CWT time-frequency representations for each temporal window. To mitigate edge effects, we considered only the spectral content inside the cone of influence. ${ }^{26}$ We computed CWT in the frequency range of $1-70 \mathrm{~Hz}$ and normalized it so that its values ranged from 0 to $1 .^{20}$

\section{Spectral entropy}

The concept of entropy originally comes from the field of thermodynamics and is related to the uncertainty of information in terms of disorder, discrepancy and diversity. ${ }^{27}$ In the context of signal processing, previous studies have used entropy to determine the degree of irregularity of a time series. ${ }^{19,28}$ In particular, a measure of entropy applied over the EEG power spectrum (i.e., spectral entropy) is an estimate of the flatness of the spectral content. ${ }^{29}$ From this point of view, spectral entropy can be considered an index of signal regularity, because a signal with a large range of spectral components (e.g., white noise) has a flat power spectral density and, therefore, high values of spectral entropy (close to 1). On the other hand, a signal with few spectral components (e.g., a pure sinusoidal wave) yields minimum spectral entropy values (close to 0 ).

We are aware that other, more traditional methods of characterizing the spectrum of a signal exist. In this respect, relative and absolute power are probably the most common measures for characterizing the power spectral density of EEG. ${ }^{27,29}$ However, they provide an estimate of the amplitude (i.e., power) of the signal on specific neural oscillation or fre- quency bands, leaving aside the interrelation between frequency bands. This relationship (or ratio) between frequencies can be directly measured using spectral entropy, because the flatness of the spectrum is a measure of the degree of equality between different frequency components.

In the present study, we calculated spectral entropy from the normalized CWT, which is sometimes called "wavelet entropy." ${ }^{29}$ Unlike its conventional variant calculated directly from the power spectral density, this method of calculating spectral entropy provides the additional advantage of analyzing the entropy variation of a signal over time. Thus, time-dependent wavelet-based spectral entropy is defined as follows:

$$
S E(t)=-\frac{1}{\log (M)} \cdot \sum_{f} W S(t, f) \cdot \log [W S(t, f)]
$$

where $S E$ is spectral entropy and WS is the wavelet across the spectrum that summarizes the distribution of the signal energy.

After we computed spectral entropy, we averaged it in the time domain in 2 windows: baseline (from $300 \mathrm{~ms}$ before the stimulus to the stimulus onset) and response (150 ms to $450 \mathrm{~ms}$ from stimulus onset). Spectral entropy was computed for each participant and window and then averaged, following the single trial approach. Spectral entropy modulation was defined as the spectral entropy change from baseline to the response window.

\section{Structural connectivity}

We based the assessment of structural connectivity data on fractional anisotropy values, using DTI and graph theory (Appendix 1, Figure S1, available at jpn.ca/190032-a1). This followed the same steps as our previous work. ${ }^{10}$ Essentially, after we acquired magnetic resonance images in a $3 \mathrm{~T}$ unit, we extracted a connectivity matrix from each participant based on cortical parcellation (FreeSurfer; https://surfer.nmr.mgh. harvard.edu) and fractional anisotropy, allowing a fractional anisotropy value to be ascribed to the connections between each pair of the 84 cortical regions. From this matrix, the small-world index (the ratio between coefficient clustering and path length after normalizing using surrogate data) and connectivity strength, we calculated an estimate of the mean number of connections for each region (Appendix 1, Figure S1).

\section{Statistical analysis}

We compared age and sex distribution between groups using analysis of variance and $\chi^{2}$ tests.

Our analyses yielded a spectral entropy value per sensor, likely correlated among sensors. To confirm collinearity, we explored that correlation using Pearson coefficients and found that spectral entropy modulation values were highly correlated among sensors $(0.329<r<0.790, p<0.001$, in all cases). Thus, we reduced spectral entropy sensor data using a factor analysis (without rotation), saving individual factor scores for further calculations. Given the number of sensors, for this calculation we used all 127 healthy controls available in our database from whom EEGs were 
obtained and processed using exactly the same conditions (including the 48 control participants matched to the patient groups). Thus, we performed this factor analysis on EEG data from 235 participants, of whom 156 were included in further analyses.

Because spectral entropy modulation is the difference of spectral entropy values between the response and pre-stimulus windows, we considered it relevant to compare spectral entropy values between groups at the pre-stimulus window. To do this, we followed the same procedure, introducing the pre-stimulus spectral entropy values in a factor analysis and saving the factor scores.

To address specificity and chronicity issues, we compared factor scores from the factor analysis (pre-stimulus and modulation spectral entropy) between groups using a general linear model with age as a covariate. We assessed the effect size of the differences as $\eta^{2}$. To facilitate the interpretability of spectral entropy modulation differences, we also calculated median frequency (defined as the frequency that split the spectral power in half) and differences at each sensor (i.e., median frequency modulation) and compared these values between groups.

To assess possible effects of antipsychotic treatment, we calculated correlation coefficients (Pearson $r$ ) between a participant's current antipsychotic dose and spectral entropy modulation factor scores and then compared these factor scores between patients with bipolar disorder who were receiving antipsychotics and those who were not. To study the possible effects of other pharmacological treatments, we compared the spectral entropy pre-stimulus and modulation factor scores between patients with bipolar disorder who were receiving lithium or not, and between patients (schizophrenia and bipolar disorder) who were receiving or not receiving antidepressants or benzodiazepines.

To study the possible effects of structural connectivity on spectral entropy modulation, we followed 2 steps. First, we compared small-world index and connectivity strength between groups using a multivariate general linear model, with age as a covariate. Then, we introduced these graph parameters into a multivariate stepwise regression model as possible predictors of spectral entropy modulation.

Finally, we assessed the possible clinical and cognitive correlates of spectral entropy modulation using regression models with factor scores of spectral entropy modulation as predictive factors of scores on the Positive and Negative Syndrome Scale and the Brief Assessment of Cognition in Schizophrenia separately in patients with schizophrenia and patients with bipolar disorder.

\section{Results}

We found no significant differences in sex distribution among the groups $\left(\chi_{3}^{2}=0.577 ; p=0.90\right)$.

Patients with first-episode schizophrenia were significantly younger, but age was not significantly different among the chronic schizophrenia, bipolar disorder and control groups (Table 1). Illness duration was significantly shorter in patients with first-episode schizophrenia, but did not differ between patients with chronic schizophrenia and patients with bipolar disorder.

All patients with schizophrenia and 17 patients with bipolar disorder were receiving stable doses of atypical antipsychotics. Twelve patients with bipolar disorder had not been receiving antipsychotic treatment for longer than 1 year and had received it only occasionally in the past, never for more than 2 weeks. Other concomitant medications are shown in Table 1.

Patients with bipolar disorder were receiving significantly smaller doses of antipsychotics than the 2 schizophrenia groups. We found no significant between-group differences in years of education for participants or their parents.

Scores on the Positive and Negative Syndrome Scale were significantly lower in patients with bipolar disorder than in patients with chronic or first-episode schizophrenia.

\section{Modulation and pre-stimulus spectral entropy differences}

Factor analysis of spectral entropy modulation revealed that the first factor explained $56.3 \%$ of the common variance (eigenvalue $=17.77)$. All sensors contributed positively to this factor (coefficients from 0.680 to 0.812; Appendix 1, Table S1, scree plot in Figure S2).

Therefore, we used a univariate general linear model to assess between-group differences (chronic and first-episode schizophrenia, bipolar disorder, healthy controls) for spectral entropy modulation. This analysis revealed a significant effect of diagnostic group $\left(F_{3}=5.752 ; p<0.001 ; \eta^{2}=0.106\right.$; a medium effect size). The effects of age $\left(F_{1}=0.459 ; p=0.50\right)$ and group $\times$ age interaction $\left(F_{1}=1.053 ; p=0.31\right)$ on spectral entropy modulation were not significant. Post-hoc comparisons revealed a significantly smaller spectral entropy modulation (i.e., a smaller spectral entropy decrease from baseline to response) in patients with chronic or first-episode schizophrenia and in patients with bipolar disorder compared with controls, but no significant differences between patient groups (Table 1 and Fig. 1). The spatial distribution revealed a large decrease in spectral entropy values over frontocentral sensors in the control and patient groups, spatially smaller in the patient groups (Fig. 2). For illustrative purposes, 95\% confidence intervals for spectral entropy modulation values in the midline, frontal and parietal electrodes per group are shown in Figure 3.

Similarly, factor analysis of pre-stimulus spectral entropy values revealed that a single factor explained $71.28 \%$ of prestimulus spectral entropy variance (eigenvalue $=20.67$ ), with all the sensors contributing positively $(0.64<r<0.911)$. The general linear model revealed no significant effect of group for pre-stimulus spectral entropy $\left(F_{3}=0.46 ; p=0.70\right)$.

The spectral entropy modulation differences were accompanied by a widespread significant decrease in median frequency from baseline to response, indicating a significant slowing of EEG that was significantly larger in the control group than in the patient groups, but not significantly different among patient groups (Appendix 1, Table S2). The spatial distribution of median frequency modulation showed a large decrease in the frontocentral regions in all groups, more widespread in the control group (Fig. 2). 


\section{Treatment effects}

Spectral entropy modulation was unrelated to illness duration $(r=0.15, p=0.24)$ or antipsychotic dose $(r=-0.06, p=$ 0.54; Fig. 4).

Comparison of spectral entropy modulation between patients with bipolar disorder receiving antipsychotic treatment (mean \pm standard deviation $0.465 \pm 0.639)$ or not $(0.540$ $\pm 0.478)$ showed no significant differences $(U=78 ; Z=-0$ to 493; $p=0.65$; Fig. 4). We found no significant differences for pre-stimulus spectral entropy between patients with bipolar disorder receiving antipsychotic treatment $(-0.11 \pm 1.05)$ or not $(0.16 \pm 0.85 ; U=75 ; Z=-0.64 ; p=0.52)$. The association between antipsychotic doses and pre-stimulus spectral entropy was not significant $(r=-0.007 ; p=0.95)$.

Similarly, we found no significant differences in spectral entropy modulation between patients with bipolar disorder receiving lithium or not, and between patients receiving benzodiazepines or antidepressants or not (Appendix 1, Fig. S3 and Table S3). Mean spectral entropy modulation values were lower (suggesting a more normal modulation) in treated patients. These treatments did not affect pre-stimulus spectral entropy values.

\section{Structural connectivity effects}

Structural small-world index and connectivity strength values were not significantly related to age $(F=0.768$ and 0.005 , respectively; $p=$ not significant). Group was associated with structural small-world index at a trend level $\left(F_{3}=\right.$ $2.419 ; p=0.07)$ and significantly associated with connectivity strength $\left(F_{3}=6.760 ; p<0.001\right)$. Pairwise comparisons showed significantly lower structural small-world index values in patients with chronic schizophrenia and patients

Table 1: Demographic, clinical, cognitive and EEG characteristics for each study group*

\begin{tabular}{|c|c|c|c|c|}
\hline Characteristic & $\begin{array}{l}\text { Chronic schizophrenia } \\
\qquad(n=48)\end{array}$ & $\begin{array}{l}\text { First-episode schizophrenia } \\
\qquad(n=31)\end{array}$ & $\begin{array}{l}\text { Bipolar disorder } \\
\quad(n=29)\end{array}$ & $\begin{array}{l}\text { Control } \\
(n=48)\end{array}$ \\
\hline Sex, M/F & $29 / 19$ & $19 / 12$ & $17 / 12$ & $26 / 22$ \\
\hline Age, yr & $38.93 \pm 9.49$ & $30.61 \pm 10.14 \dagger$ & $46.40 \pm 10.45$ & $41.77 \pm 8.97$ \\
\hline Illness duration, mo & $156.17 \pm 115.62$ & $23.375 \pm 58.70 \S$ & $189.21 \pm 106.04$ & NA \\
\hline Antipsychotic dose, CPZ eq, mg/d & $447.48 \pm 412.95$ & $357.40 \pm 227.11$ & $166.1 \pm 155.87 \ddagger$ & NA \\
\hline Atypical antipsychotic, $n$ & 48 & 31 & 17 & NA \\
\hline Benzodiazepine, $n$ & 20 & 9 & 7 & NA \\
\hline Antidepressant, $n$ & 12 & 4 & 6 & NA \\
\hline Lithium, $n$ & NA & NA & 17 & NA \\
\hline Anticonvulsant, $n$ & NA & NA & 5 & NA \\
\hline Education, yr & $13.71 \pm 4.09$ & $15.47 \pm 3.00$ & $15.80 \pm 1.78$ & $13.20 \pm 5.44$ \\
\hline Parental education, yr & $11.00 \pm 4.51$ & $13.21 \pm 4.17$ & $10.00 \pm 2.44$ & $9.63 \pm 3.92$ \\
\hline PANSS positive score & $11.4 \pm 4.25$ & $10.63 \pm 2.77$ & $7.36 \pm 0.83 \ddagger$ & NA \\
\hline PANSS negative score & $17.75 \pm 8.73$ & $14.83 \pm 4.22$ & $9.05 \pm 2.48 \ddagger$ & NA \\
\hline PANSS total score & $56.07 \pm 21.25$ & $47.6 \pm 11.18$ & $30.57 \pm 3.32 \ddagger$ & NA \\
\hline Young Mania Rating Scale score & $0.83 \pm 1.91$ & $0.22 \pm 0.71$ & $1.81 \pm 1.34$ & NA \\
\hline Hamilton Depression Rating Scale score & $5.77 \pm 4.29$ & $5.91 \pm 4.18$ & $4.98 \pm 3.81$ & NA \\
\hline \multicolumn{5}{|c|}{ Brief Assessment of Cognition in Schizophrenia score } \\
\hline Verbal memory & $32.75 \pm 14.87 \ddagger$ & $37.62 \pm 10.57 \ddagger$ & $36.00 \pm 9.25 \ddagger$ & $48.55 \pm 8.53$ \\
\hline Working memory & $15.97 \pm 4.85 \ddagger$ & $16.38 \pm 4.62 \ddagger$ & $17.57 \pm 4.03 \ddagger$ & $22.04 \pm 3.35$ \\
\hline Motor speed & $61.42 \pm 21.29 \ddagger$ & $55.57 \pm 15.16 \ddagger$ & $66.10 \pm 13.67 \ddagger$ & $76.90 \pm 13.79$ \\
\hline Verbal fluency & $17.37 \pm 7.04 \ddagger$ & $16.79 \pm 7.31 \ddagger$ & $19.78 \pm 7.87 \dagger$ & $27.21 \pm 7.41$ \\
\hline Performance speed & $39.76 \pm 15.30 \ddagger$ & $45.11 \pm 13.12 \ddagger$ & $41.84 \pm 12.40 \ddagger$ & $65.76 \pm 14.57$ \\
\hline Problem-solving & $16.52 \pm 4.60$ & $15.24 \pm 3.93$ & $15.94 \pm 3.30$ & $17.18 \pm 3.23$ \\
\hline MSCEIT score & $92.38 \pm 23.51 \neq$ & $101.27 \pm 18.69 \ddagger$ & $104.05 \pm 22.09 \ddagger$ & $120.61 \pm 22.27$ \\
\hline Total IQ & $95.27 \pm 14.07 \ddagger$ & $87.50 \pm 14.70 \ddagger$ & $98.31 \pm 10.23 \ddagger$ & $115.11 \pm 10.39$ \\
\hline P300 amplitude, mV & $1.04 \pm 0.61$ & $1.20 \pm 0.70$ & $1.23 \pm 0.63$ & $1.58 \pm 0.76$ \\
\hline P300 latency, ms & $423.22 \pm 95.43$ & $455.13 \pm 87.39$ & $464.69 \pm 96.11$ & $458.54 \pm 71.40$ \\
\hline Spectral entropy modulation, factor score & $0.198 \pm 0.134 \ddagger$ & $0.067 \pm 0.183 \dagger$ & $0.536 \pm 0.185 \S$ & $-0.345 \pm 0.134$ \\
\hline Structural small-world index & $\begin{array}{c}0.97 \pm 0.01 \dagger \\
(n=21)\end{array}$ & $\begin{array}{c}0.98 \pm 0.00 \\
(n=18)\end{array}$ & $\begin{aligned} 0.97 & \pm 0.01 \dagger \\
(n & =21)\end{aligned}$ & $\begin{array}{c}0.98 \pm 0.00 \\
(n=20)\end{array}$ \\
\hline Structural connectivity strength & $\begin{array}{l}0.31 \pm 0.03 \S \\
\quad(n=21)\end{array}$ & $\begin{array}{c}0.34 \pm 0.01 \\
(n=18)\end{array}$ & $\begin{array}{l}0.30 \pm 0.03 \S \\
\quad(n=21)\end{array}$ & $\begin{array}{l}0.34 \pm 0.03 \\
(n=20)\end{array}$ \\
\hline \multicolumn{5}{|c|}{$\begin{array}{l}\text { CPZ eq = chlorpromazine equivalent; EEG = electroencephalogram; IQ = intelligence quotient; MSCEIT = Meyer, Salovey and Caruso Emotional Intelligence Test; NA = not applicable; } \\
\text { PANSS = Positive and Negative Syndrome Scale. } \\
\text { *Spectral entropy modulation values correspond to factor scores from the factor analysis (see text). Findings are presented as mean } \pm \text { standard deviation, unless otherwise indicated. } \\
\text { Significant differences with respect to controls are highlighted }(\dagger p<0.05 ; \ddagger p<0.01 ; \S p<0.001) \text {. For PANSS scores, illness duration and treatment doses, differences were calculated } \\
\text { between patient groups only. }\end{array}$} \\
\hline
\end{tabular}


with bipolar disorder compared to controls but not to patients with first-episode schizophrenia, and lower structural connectivity strength values in patients with chronic schizophrenia and patients with bipolar disorder compared to controls and patients with first-episode schizophrenia (Table 1).
Structural connectivity parameters were not associated with spectral entropy modulation (Appendix 1, Figure S4). To discard a hidden relationship between structural data (assessed as small-world index or connectivity strength) and spectral entropy modulation, we calculated Pearson correlation coefficients between both types of variables. These coefficients were
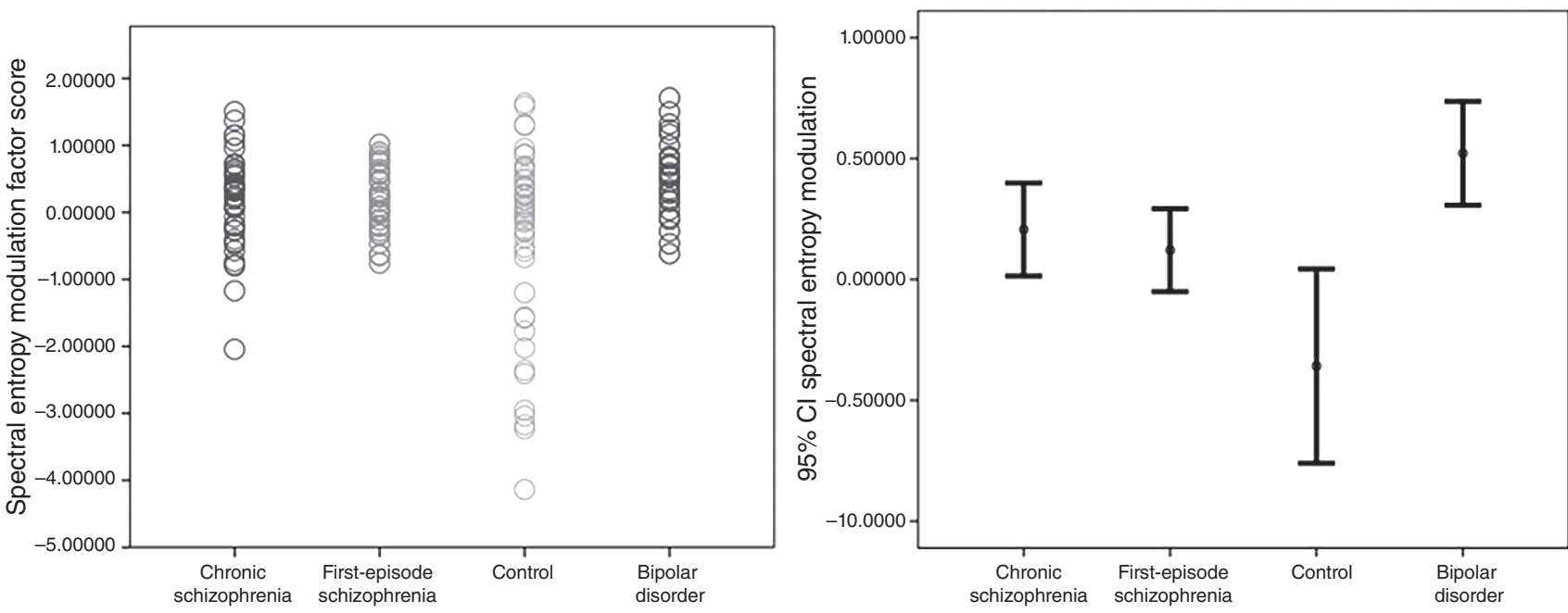

Fig. 1: Spectral entropy modulation (factor scores): individual distributions and 95\% confidence intervals (Cl).

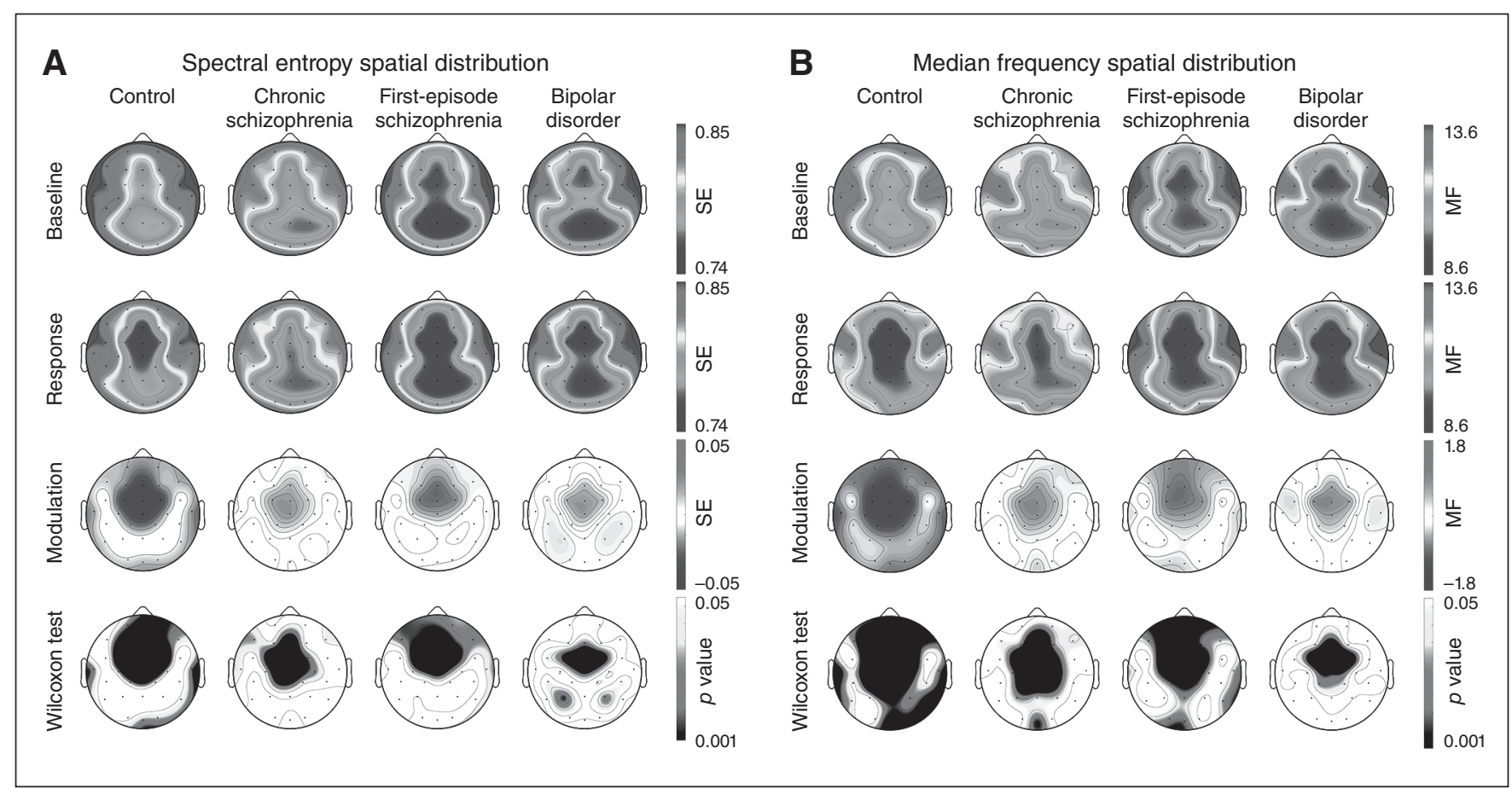

Fig. 2: Spatial distribution of spectral entropy (SE) and median frequency (MF) at baseline and response windows, and its difference (modulation) in each group, with corresponding intragroup statistical significance difference between windows (Wilcoxon test). Shading depicts the corresponding regional values in Hertz for median frequency. Between-group comparisons are shown in the text. 
not significant for the whole sample $(n=80 ; r=-0.181$ and $-0.183)$, for patients ( $n=60 ; r=-0.182$ and -0 to 112) or for patients with schizophrenia $(n=39 ; r=-0.074$ and -0.059$)$.

\section{Clinical and cognitive correlates}

In the schizophrenia group we found a significant association between spectral entropy modulation and negative symptoms $\left(F_{1,78}=5.353 ; \beta=0.270 ; p=0.02\right)$ but not positive symptoms $\left(F_{1,78}=0.170 ; p=0.681 ;\right.$ Fig. 5$)$. Thus, a smaller decrease of taskrelated spectral entropy (a task-related decrease in entropy is expected in healthy controls) was associated with higher negative symptom scores. In the same group, verbal memory $\left(R_{1,77}^{2}=0.055 ; \beta=-0.227 ; p=0.05\right)$ and working memory $\left(R_{1,77}^{2}=0.047 ; \beta=-0.217 ; p=0.08 ;\right.$ Fig. 4$)$ were associated with spectral entropy modulation. Thus, a higher task-related spectral entropy decrease was associated with better cognitive performance. In patients with bipolar disorder, spectral entropy modulation was not associated with cognition or symptoms. Spectral entropy modulation was not associated with social cognition or scores on the Hamilton Depression Rating Scale or Young Mania Rating Scale.
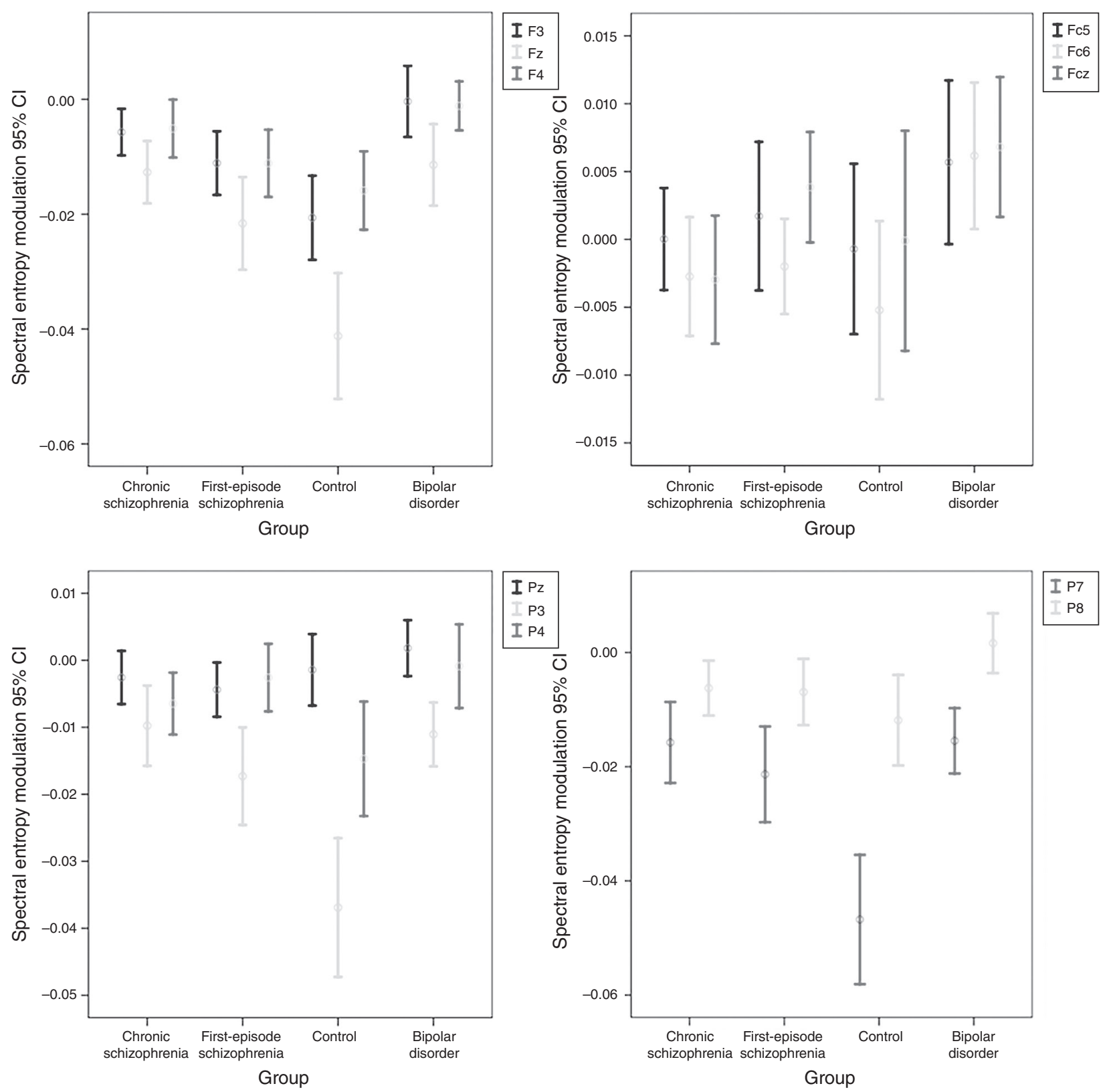

Fig. 3: Spectral entropy modulation $95 \%$ confidence intervals $(\mathrm{Cl})$ at frontal $(\mathrm{F})$, frontocentral $(\mathrm{Fc})$, and parietal $(\mathrm{P})$ electrodes. 
A database with the main findings supporting the present results is freely available (Mendeley Data DOI:0.17632/ sr56trwfhf.2)

\section{Discussion}

According to our results, a deficit in spectral entropy modulation occurs in patients with first-episode and chronic schizophrenia and euthymic patients with bipolar disorder. Although we expected significant differences in spectral entropy modulation between patients with schizophrenia and patients with bipolar disorder, our results suggest that this deficit may characterize both syndromes.

Mental functions are likely underpinned by the coordination of cerebral network activity, reflected in evolving EEG patterns. ${ }^{1,3}$ Our data suggest that deficits in neural synchronization might contribute to psychoses. Brain processing de- pends on short- and long-range interactions between neural assemblies. ${ }^{28,30,31}$ In this context, our data might indicate that abnormal cerebral processing, reflected in a decreased EEG modulation during performance of a task with a small cognitive load, could contribute to aberrant mental representations in the form of the symptoms and/or cognitive deficits of psychosis. Therefore, deficits in spectral entropy (and median frequency) modulation could represent a marker of disease related to altered function in psychotic syndromes. This claim is supported by the new replication in the present study of the spectral entropy modulation deficits in EEG described in 2 previous samples. ${ }^{6,7}$ Our results also reveal a significantly smaller median frequency modulation (i.e., a smaller slowing of the EEG) in patients with psychosis, similar to findings in previous samples, where a lower increase of $\theta$ density was found in patients with the same paradigm. ${ }^{6}$ This may suggest a lower capacity for synchronizing
A
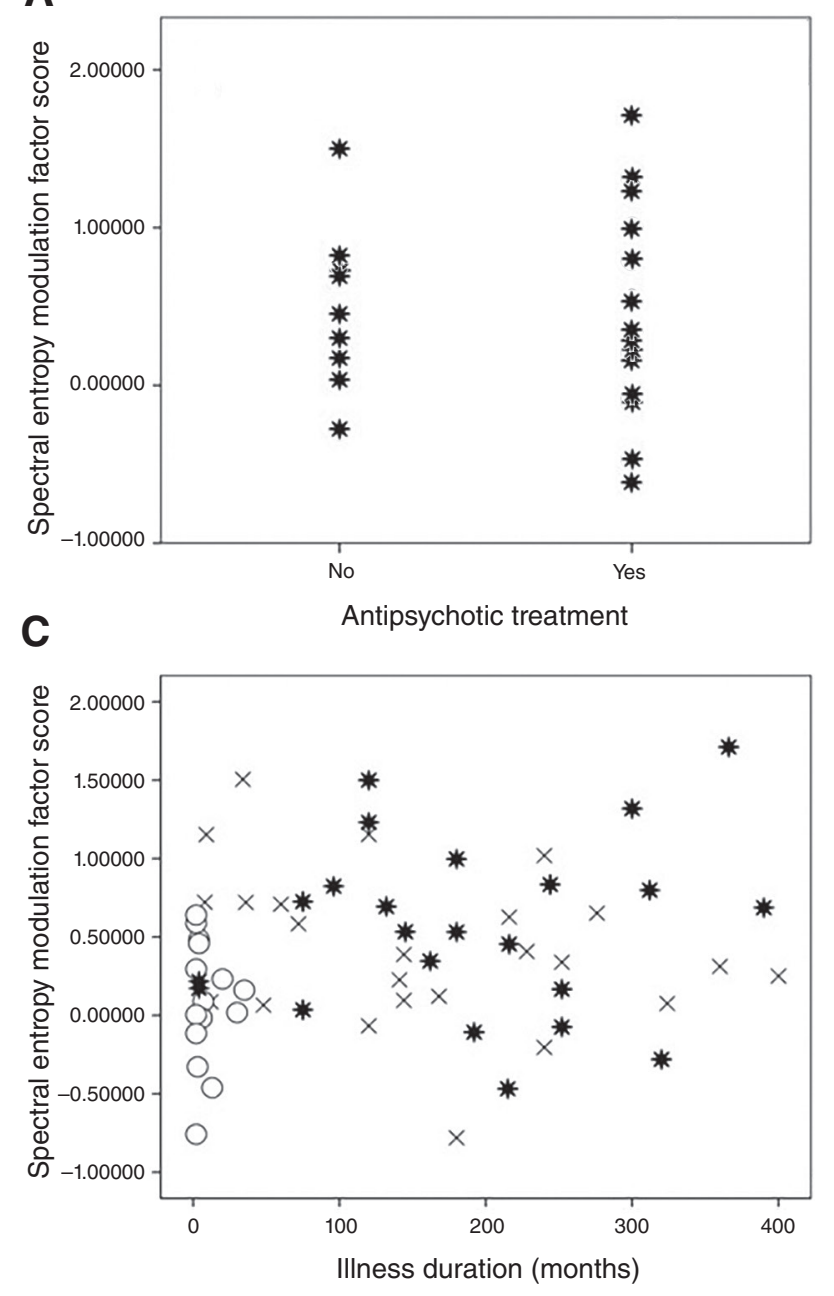

B

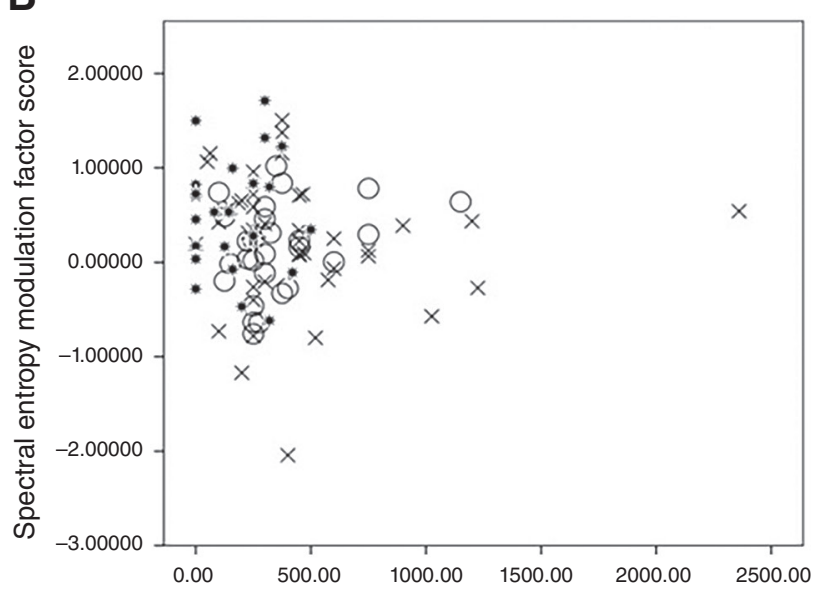

Treatment dose (chlorpromazine equivalents in $\mathrm{mg} / \mathrm{d}$ )

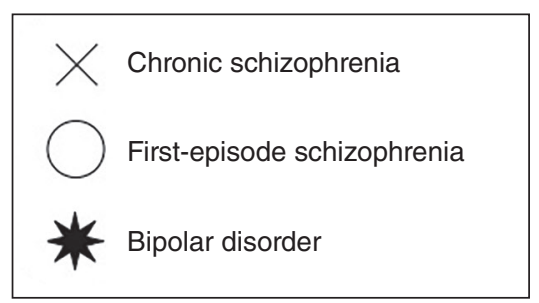

Fig. 4: Graphs showing the absence of a relation between spectral entropy modulation and treatment. (A) Spectral entropy modulation values in patients with bipolar disorder receiving antipsychotic treatment or not. (B) Association between current treatment antipsychotic doses and spectral entropy modulation. (C) Association between illness duration and spectral entropy modulation. 
distant regions in patients with psychosis, given the proposed role for $\theta$ activity in long-range synchronization. ${ }^{28}$

In this context, it is important to note that the P300 task does not directly assess cognition, because its cognitive load is rather small. Thus, our data do not necessarily indicate that the described deficit in spectral entropy modulation is a causal mechanism in the cognitive deficits in schizophrenia. Instead, a more conservative interpretation of our data is that global brain activity in schizophrenia and bipolar disorder shows a significant deficit in expected task-related modulation, which may hamper cognitive performance and mental representations under more demanding conditions.

We found no significant differences in pre-stimulus spectral entropy, similar to previous reports, ${ }^{6,7}$ supporting the concept that the primary deficit in patients relates to modulation itself. All sensors contributed positively to the first factor of the factor analyses on pre-stimulus and modulation spectral entropy, indicating a similar global pattern for both baseline EEG and its task modulation. However, we cannot discount the possibility that some regions with a slightly different spectral entropy baseline might have gone unnoticed in the factor analysis. Visual inspection of the prestimulus spectral entropy and median frequency showed a slower pre-stimulus EEG at the midline that might have corresponded to the default mode network, whose resting hyperactivity has been described in schizophrenia and bipolar disorder ${ }^{32,33}$ and may hamper task-related activation. ${ }^{34}$ In other words, the global deficit of spectral entropy

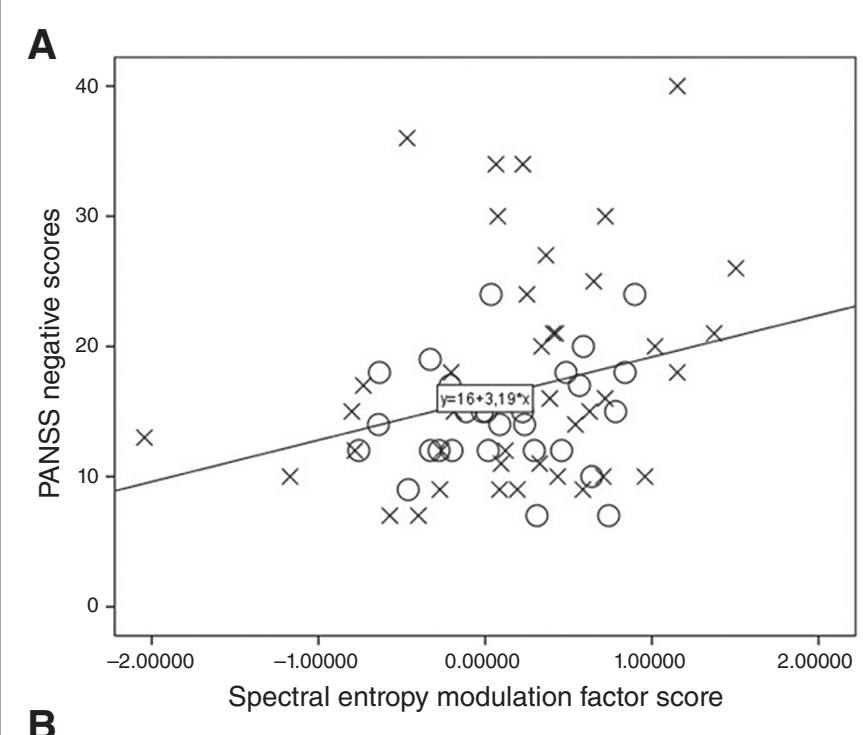

B

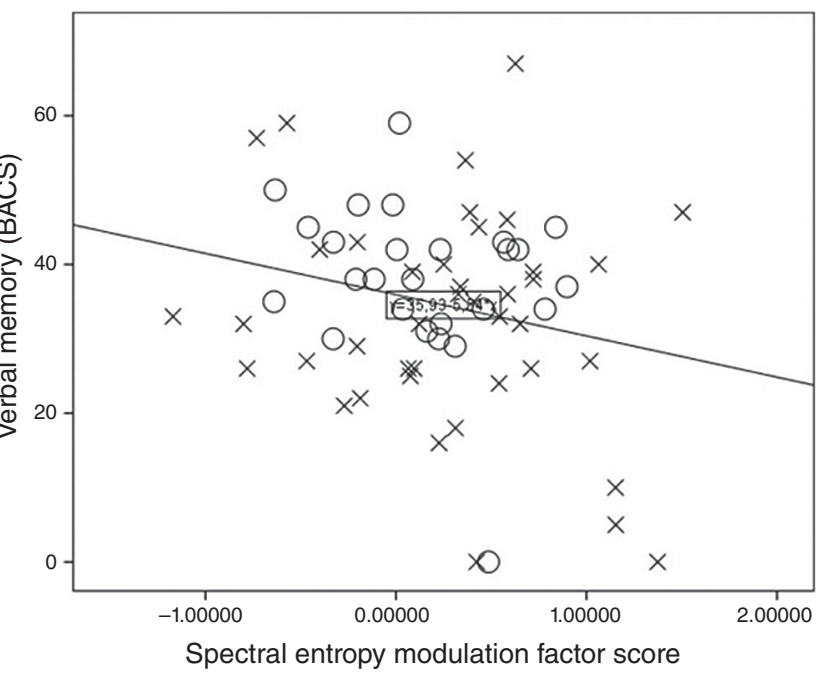

C

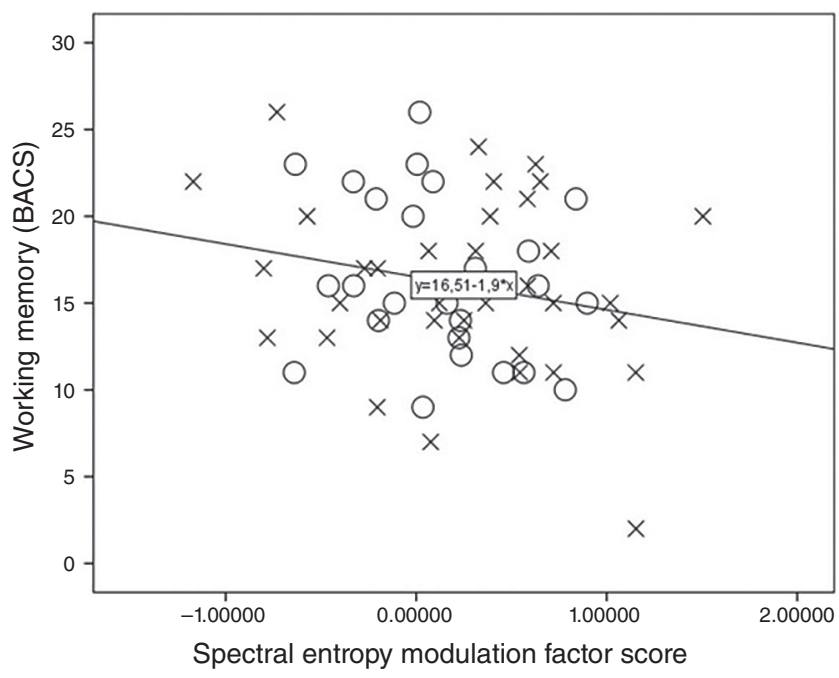

Fig. 5: Scatterplots showing the relationship between spectral entropy modulation and (A) negative symptom scores and performance in (B) verbal and $(C)$ working memory in patients with schizophrenia $(p<0.05$; see text). BACS = Brief Assessment of Cognition in Schizophrenia; PANSS $=$ Positive and Negative Syndrome Scale. 
modulation might be related to regional baseline alterations in the midline.

As for the possible biological substrates for a deficit in spectral entropy modulation, a role can be speculated for $\gamma$-aminobutyric acid (GABA) deficits in psychoses, because inhibitory transmission is key in selecting the neural assemblies that underlie task-related patterns of cortical activation. ${ }^{35}$ Alterations in GABA transmission are found in schizophrenia ${ }^{36}$ and mood disorders. ${ }^{37}$ Such alterations could contribute to a deficit in spectral entropy modulation in psychoses by decreasing the capacity to select adequate neural assemblies, at least in some patients. In support of this possibility, a larger amount of "noise power" (a measurement of background EEG activity unrelated to the task being performed) at the $\mathrm{Fz}$ electrode was inversely associated with spectral entropy modulation in a previous report. ${ }^{38}$ In other words, a lower inhibitory function might reasonably increase the amount of noise power (i.e., background activity), which could decrease the reserve for taskrelated modulation of the EEG. Similarly, large functional connectivity strength at baseline in schizophrenia (likely reflecting a hyperactive basal state) predicted smaller spectral entropy modulation. ${ }^{8}$ As well, using EEG and source analyses, we have reported a deficit in activation of the anterior cingulate and lateral and medial prefrontal regions during the same P300 task in 2 completely different samples, ${ }^{39,40}$ where GABA concentrations were associated with task-related activity modulation. ${ }^{41}$ In first-episode schizophrenia, anterior cingulate GABA levels were correlated with task-related activation. ${ }^{42}$ Taken together, these data might support a role for a GABA deficit in the frontal and cingulate regions in the deficits in global spectral entropy modulation in our sample. However, this speculation should be demonstrated using adequate tools allowing for the assessment of GABA function in vivo, such as transcranial magnetic stimulation.

A common deficit in spectral entropy modulation across groups of patients does not imply a single substrate for this deficit within or across diagnoses. Because a considerable degree of biological heterogeneity is likely in psychoses, ${ }^{43}$ different neural alterations could contribute to deficits in spectral entropy modulation. The assessment of the possible underpinnings of deficits in spectral entropy modulation was beyond our reach for the present report, but the genetic heterogeneity of psychosis might suggest that several neural alterations may contribute to that deficit in different psychotic subpopulations. Indeed, recent data support the concept that only a subset of patients with schizophrenia or bipolar disorder is characterized by GABA transmission deficits. ${ }^{44}$

In our schizophrenia sample, a smaller spectral entropy modulation was associated with worse performance in memory tasks. This association would suggest that in schizophrenia, a decreased capacity for modifying the underlying functional connections during the P300 task might also hamper the configuration of neural assemblies underlying mnemonic representations. The lack of a similar association in the bipolar disorder group might be secondary to the smaller size of this group.
In the present study, deficits in spectral entropy modulation were associated with negative symptoms in patients with schizophrenia, similar to a previous report. ${ }^{7}$ This could be consistent with a detachment from the environment associated with (and perhaps secondary to) a lower synchronization among cerebral areas. This could lead to a decreased synthesis of relevant cognitive and emotional aspects of mental life, which could be observed as negative symptoms as proposed in phenomenological accounts of psychoses. ${ }^{45}$ The low level of affective or psychotic symptoms in the bipolar group precluded the drawing of conclusions about the relationship between symptoms and spectral entropy modulation in this group.

It was remarkable that deficits in spectral entropy modulation were independent of treatment. Current antipsychotic doses were unrelated to spectral entropy modulation, and patients with bipolar disorder showed similar spectral entropy values whether they received antipsychotic treatment or not. Similarly, treatment with lithium, benzodiazepines and antidepressants did not produce the observed deficits in spectral entropy modulation, either in the bipolar group or in all participants considered together. This was consistent with the absence of significant effect of a single dose of haloperidol on spectral entropy in healthy controls. ${ }^{6}$

Structural architecture (based on fractional anisotropy, and thus reflecting white matter integrity) did not predict spectral entropy modulation. In this sample, significant structural deficits were observed only in patients with chronic schizophrenia, but small-world index architecture did not differ between patients with chronic and first-episode schizophrenia. A metaanalysis supported structural connectivity deficits in firstepisode schizophrenia. ${ }^{46}$ Taken together, these data may suggest a dissociation between functional dysconnectivity and structural architecture in schizophrenia, which may contribute to the proposed heterogeneity in this disorder. However, in a previous report, we found that fractional anisotropy in specific structural connections might contribute to EEG modulation $^{39}$; this should be studied further.

\section{Limitations}

Our study had some limitations. Because patients with bipolar disorder were in a euthymic state, we could not assess the possible correlates between affective symptoms and spectral entropy modulation. However, despite their euthymia, patients with bipolar disorder showed a significant deficit in spectral entropy modulation, supporting its role as a trait marker. We lacked a group of patients with first-episode bipolar disorder that could have allowed us to assess chronicity effects in this disorder. Moreover, the lack of a neuroleptic-free schizophrenia group limited our capacity to assess the contribution of treatment to spectral entropy modulation in schizophrenia. However, the absence of spectral entropy differences between patients with bipolar disorder exposed to antipsychotics or not argues against a major role for antipsychotics in deficits in spectral entropy modulation. Finally, the effects of psychoactive drugs may influence EEG modulation, although our patients were not actively consuming such substances. 


\section{Conclusion}

This was the third sample in which we found cognitionrelated deficits in spectral entropy modulation in schizophrenia, including a first-episode subgroup, and we had similar findings in euthymic patients with bipolar disorder. These deficits did not appear to be an effect of treatment or chronicity and were related in the schizophrenia group to cognitive deficits and negative symptoms.

Acknowledgements: This work has been supported by the following grants: the "Instituto de Salud Carlos III" (PI15/00299 and PI18/00178) the" Gerencia Regional de Salud de Castilla y León" (GRS GRS 1485/A/17 and GRS 1721/A/18); the 'Ministerio de Economía, Industria y Competitividad" and FEDER (TEC201453196-R), "Consejería de Educación de la Junta de Castilla y León" (VA059U13 and VA057P17); and predoctoral fellowship to A. Lubeiro ("Consejería de Educación Junta de Castilla y León"). J. Gomez-Pilar is supported by a predoctoral fellowship from the University of Valladolid. P. Holgado-Madera is supported by a predoctoral fellowship from the Consejería de Educación Junta de Castilla y León and European Social Fund. P. Núñez is supported by a predoctoral scholarship 'Ayuda para contratos predoctorales para la Formación de Profesorado Universitario (FPU)' from the 'Ministerio de Educación, Cultura y Deporte' (FPU17/00850). J. Fernández-Sevillano is supported by the Predoctoral Training Programme of the Education Department of the Basque Government.

Affiliations: From the Psychiatry Department, School of Medicine, University of Valladolid, Valladolid, Spain (Molina, Lubeiro); the Psychiatry Service, Clinical Hospital of Valladolid, Valladolid, Spain (Molina, Martín-Santiago); the Neurosciences Institute of Castilla y León (INCYL), University of Salamanca, Salamanca, Spain (Molina); the Imaging Processing Laboratory, University of Valladolid, Valladolid, Spain (de Luis Garcia); the Biomedical Engineering Group, University of Valladolid, Valladolid, Spain (Gomez-Pilar, Núñez); the Neurophysiology Service, Clinical Hospital of Valladolid, Valladolid, Spain (Iglesias-Tejedor); the Psychiatry Service, Doce de Octubre University Hospital, Madrid, Spain (Holgado-Madera, SanzFuentenebro); the Psychiatry Service, Cruces Hospital, Bilbao, Spain (Segarra-Echeverría, Recio-Barbero); and the Psychiatry Service, Santiago Apostol Hospital, Vitoria, Spain (Haidar, Fernández-Sevillano).

Competing interests: None declared.

Contributors: V. Molina and R. de Luis Garcia designed the study. V. Molina, A. Lubeiro, O. Martín-Santiago, M. Iglesias-Tejedor, P. Holgado-Madera, R. Segarra-Echeverría, M. Recio-Barbero, P. Núñez, M. Haidar, J. Fernández-Sevillano and J. Sanz-Fuentenebro acquired the data, which V. Molina, R. de Luis Garcia, J. Gomez-Pilar and P. Núñez analyzed. V. Molina and A. Lubeiro wrote the article, which all authors reviewed. All authors approved the final version for publication and can certify that no other individuals not listed as authors have made substantial contributions to the paper.

\section{References}

1. Varela F, Lachaux JP, Rodriguez E, et al. The brainweb: phase synchronization and large-scale integration. Nat Rev Neurosci 2001; 2:229-39

2. Buzsaki G, Draguhn A. Neuronal oscillations in cortical networks. Science 2004;304:1926-9.

3. Dehaene S, Changeux JP. Experimental and theoretical approaches to conscious processing. Neuron 2011;70:200-27.

4. Uhlhaas PJ, Singer W. Abnormal neural oscillations and synchrony in schizophrenia. Nat Rev Neurosci 2010;11:100-13.

5. Duff BJ, Macritchie KA, Moorhead TW, et al. Human brain imaging studies of DISC1 in schizophrenia, bipolar disorder and depression: a systematic review. Schizophr Res 2013;147:1-13.
6. Bachiller A, Diez A, Suazo V, et al. Decreased spectral entropy modulation in patients with schizophrenia during a P300 task. Eur Arch Psychiatry Clin Neurosci 2014;264:533-43.

7. Molina V, Bachiller A, Gomez-Pilar J, et al. Deficit of entropy modulation of the EEG in schizophrenia associated to cognitive performance and symptoms. A replication study. Schizophr Res 2018;195:334-42.

8. Gomez-Pilar J, de Luis-Garcia R, Lubeiro A, et al. Deficits of entropy modulation in schizophrenia are predicted by functional connectivity strength in the theta band and structural clustering. Neuroimage Clin 2018;18:382-9.

9. Ellison-Wright I, Bullmore E. Meta-analysis of diffusion tensor imaging studies in schizophrenia. Schizophr Res 2009;108:3-10.

10. Gomez-Pilar J, de Luis-Garcia R, Lubeiro A, et al. Relations between structural and EEG-based graph metrics in healthy controls and schizophrenia patients. Hum Brain Mapp 2018;39:3152-65.

11. Adachi Y, Osada T, Sporns O, et al. Functional connectivity between anatomically unconnected areas is shaped by collective network-level effects in the macaque cortex. Cereb Cortex 2012; 22:1586-92.

12. Hamilton M. A rating scale for depression. J Neurol Neurosurg Psychiatry. 1960;23:56-62.

13. Young RC, Biggs JT, Ziegler VE, et al. A rating scale for mania: reliability, validity and sensitivity. Br I Psychiatry 1978;133:429-35.

14. Kay SR, Fiszbein A, Opler LA. The positive and negative syndrome scale (PANSS) for schizophrenia. Schizophr Bull 1987;13:261-76.

15. Wechsler D. Wechsler adult intelligence scale. 3rd ed. San Antonio (TX): Psychological Corporation; 1997.

16. Chelune GJ, Baer RA. Developmental norms for the Wisconsin Card Sorting Test. J Clin Exper Neuropsychol 1986;8:219-28.

17. Segarra N, Bernardo M, Gutierrez F, et al. Spanish validation of the Brief Assessment in Cognition in Schizophrenia (BACS) in patients with schizophrenia and healthy controls. Eur Psychiatry 2011;26:69-73.

18. Mayer JD, Salovey P, Caruso DR, Sitarenios G. Measuring emotional intelligence with the MSCEIT V2.0: work-life strategies. Emotion 2003;3:97-105.

19. Bledowski C, Prvulovic D, Hoechstetter K, et al. Localizing P300 generators in visual target and distractor processing: a combined event-related potential and functional magnetic resonance imaging study. J Neurosci 2004;24:9353-60.

20. Gómez-Pilar J, Poza J, Bachiller A, et al. Neural network reorganization analysis during an auditory oddball task in schizophrenia using wavelet entropy. Entropy (Basel) 2015;17:5241-56.

21. Bachiller A, Poza J, Gomez C, et al. A comparative study of eventrelated coupling patterns during an auditory oddball task in schizophrenia. J Neural Eng 2015;12:016007.

22. Delorme A, Makeig S. EEGLAB: an open source toolbox for analysis of single-trial EEG dynamics including independent component analysis. J Neurosci Methods 2004;134:9-21.

23. Poza J, Gomez C, Gutierrez MT, et al. Effects of a multi-sensory environment on brain-injured patients: assessment of spectral patterns. Med Eng Phys 2013;35:365-75.

24. Bruna R, Poza J, Gomez C, et al. Analysis of spontaneous MEG activity in mild cognitive impairment and Alzheimer's disease using spectral entropies and statistical complexity measures. J Neural Eng 2012;9:036007.

25. Nunez P, Poza J, Bachiller A, et al. Exploring non-stationarity patterns in schizophrenia: neural reorganization abnormalities in the alpha band. J Neural Eng 2017;14:046001.

26. Torrence C, Compo G. A practical guide to wavelet analysis. Bull Am Meteorol Soc 1998;79:61-8.

27. Scheeringa R, Fries P, Petersson KM, et al. Neuronal dynamics underlying high- and low-frequency EEG oscillations contribute independently to the human BOLD signal. Neuron 2011;69:572-83.

28. Finelli LA, Achermann P, Borbely AA. Individual "fingerprints" in human sleep EEG topography. Neuropsychopharmacology 2001; 25:S57-62.

29. von Stein A, Chiang C, Konig P. Top-down processing mediated by interareal synchronization. Proc Natl Acad Sci U S A 2000;97:14748-53.

30. Womelsdorf T, Schoffelen JM, Oostenveld R, et al. Modulation of neuronal interactions through neuronal synchronization. Science 2007;316:1609-12.

31. Kopell N, Ermentrout GB, Whittington MA, et al. Gamma rhythms and beta rhythms have different synchronization properties. Proc Natl Acad Sci U S A 2000;97:1867-72. 
32. Pomarol-Clotet E, Moro N, Sarro S, et al. Failure of de-activation in the medial frontal cortex in mania: evidence for default mode network dysfunction in the disorder. World J Biol Psychiatry 2012; 13:616-26.

33. Pomarol-Clotet E, Salvador R, Sarro S, et al. Failure to deactivate in the prefrontal cortex in schizophrenia: dysfunction of the default mode network? Psychol Med 2008;38:1185-93.

34. Whitfield-Gabrieli S, Thermenos HW, Milanovic S, et al. Hyperactivity and hyperconnectivity of the default network in schizophrenia and in first-degree relatives of persons with schizophrenia. Proc Natl Acad Sci U S A 2009;106:1279-84.

35. Buzsáki G. Diversity of cortical functions is provided by inhibition. In: Rhythms of the brain. New York: Oxford University Press; 2006: cycle 3.

36. Gonzalez-Burgos G, Fish KN, Lewis DA. GABA neuron alterations, cortical circuit dysfunction and cognitive deficits in schizophrenia. Neural Plast 2011;2011:723184.

37. Fee C, Banasr M, Sibille E. Somatostatin-positive gamma-aminobutyric acid interneuron deficits in depression: cortical microcircuit and therapeutic perspectives. Biol Psychiatry 2017;82:549-59.

38. Molina V, Bachiller A, Suazo V, et al. Noise power associated with decreased task-induced variability of brain electrical activity in schizophrenia. Eur Arch Psychiatry Clin Neurosci 2016;266:55-61.

39. Molina V, Bachiller A, de Luis R, et al. Topography of activation deficits in schizophrenia during P300 task related to cognition and structural connectivity. Eur Arch Psychiatry Clin Neurosci 2019; 269:419-28.

40. Bachiller A, Romero S, Molina V, et al. Auditory P3a and P3b neural generators in schizophrenia: an adaptive sLORETA P300 localization approach. Schizophr Res 2015;169:318-25.

41. Northoff G, Walter M, Schulte RF, et al. GABA concentrations in the human anterior cingulate cortex predict negative BOLD responses in fMRI. Nat Neurosci 2007;10:1515-7.

42. Overbeek G, Gawne TJ, Reid MA, et al. Relationship between cortical excitation and inhibition and task-induced activation and deactivation: a combined magnetic resonance spectroscopy and functional magnetic resonance imaging study at $7 \mathrm{~T}$ in first-episode psychosis. Biol Psychiatry Cogn Neurosci Neuroimaging 2019;4:121-30.

43. Wolfers T, Doan NT, Kaufmann T, et al. Mapping the heterogeneous phenotype of schizophrenia and bipolar disorder using normative models. JAMA Psychiatry 2018;75:1146-55

44. Volk DW, Sampson AR, Zhang Y, et al. Cortical GABA markers identify a molecular subtype of psychotic and bipolar disorders. Psychol Med 2016;46:2501-12.

45. Saas LA, Parnas J. Explaining schizophrenia: the relevance of phenomenology. In: Chung MC, Fulford B, Graham G, editors. Reconceiving schizophrenia. New York: Oxford University Press; 2007: pp. 270-294.

46. Yao L, Lui S, Liao Y, et al. White matter deficits in first episode schizophrenia: an activation likelihood estimation meta-analysis. Prog Neuropsychopharmacol Biol Psychiatry 2013;45:100-6.

\section{Journal of Psychiatry et) Neuroscience}

\section{Call for submissions}

Have expertise treating patients with psychiatric disorders? Share it with clinicians in a Psychopharmacology for the Clinician column. Columns are 650 words and include a clinical vignette showcasing a topic of interest. Cases should have a level of complexity or novelty that will help clinicians make treatment decisions in situations that are not routine, or where new evidence is available but not widely known.

\section{Why write for JPN?}

- JPN is the highest ranking open access journal in biological psychiatry

- Psychopharmacology for the Clinician columns are the most downloaded feature of JPN and archives are available indefinitely on jpn.ca and in PubMed Central

Submit columns online at https://mc.manuscriptcentral.com/jpn. View previous columns at https://jpn.ca/psychopharmacology-for-the-clinician/ 